Article

\title{
Noether Symmetries Quantization and Superintegrability of Biological Models
}

\author{
Maria Clara Nucci * and Giampaolo Sanchini \\ Dipartimento di Matematica e Informatica, Università degli Studi di Perugia, 06123 Perugia, Italy; \\ giampaolo.sanchini@istruzione.it \\ * Correspondence: nucci@unipg.it
}

Academic Editor: Roman M. Cherniha

Received: 17 October 2016; Accepted: 12 December 2016; Published: 20 December 2016

\begin{abstract}
It is shown that quantization and superintegrability are not concepts that are inherent to classical Physics alone. Indeed, one may quantize and also detect superintegrability of biological models by means of Noether symmetries. We exemplify the method by using a mathematical model that was proposed by Basener and Ross (2005), and that describes the dynamics of growth and sudden decrease in the population of Easter Island.
\end{abstract}

Keywords: Lie symmetries; Jacobi last multiplier; Lagrangians; Noether symmetries; classical quantization; superintegrability; Easter Island population

\section{Introduction}

In a review to honor the 50th Anniversary Year of the Journal of Theoretical Biology [1], one reads:

"It is frequently claimed that- like Newton's invention of calculus-biological theory will require 'new mathematics'.... There are, however, many areas of mathematics that have been neglected by theoretical biology that could prove to be of great value. Einstein's work on general relativity, for instance, made good use of mathematical ideas, in particular differential geometry that had previously been developed with completely different motivation. More likely than not, the formal structures have been set forth in some context, and await their discovery and subsequent development in representing biological theory."

Since many mathematical tools used in physics have also been used in biology with different success, we present a somewhat forgotten and neglected tool, a tool that in one of its outcomes, Noether symmetries, helped Einstein and Klein in their quarrel with Hilbert about the energy-momentum conservation of general relativity theory [2]. This tool is Lie continuous symmetries, which yield conservation laws, calculus of variation setting, and ultimately quantization.

The applications of Lie symmetries to various biological models have already been shown to either provide more accurate predictions [3] or implement [4-8] the usual techniques related to qualitative and numerical analysis, which are common tools for any mathematical biologist.

We would like to stir up some controversy with the purpose of making both mathematicians and biologists ponder over some missed opportunities [9].

In [10], a mathematical model that describes the dynamics of growth and sudden decrease in the population of Easter Island was proposed. The model is a nonlinear system of two first-order ordinary differential equations, i.e.,

$$
\begin{aligned}
& \dot{w}_{1}=c w_{1}\left(1-\frac{w_{1}}{K}\right)-h w_{2}, \\
& \dot{w}_{2}=a w_{2}\left(1-\frac{w_{2}}{w_{1}}\right),
\end{aligned}
$$


where $w_{1}$ is the amount of resources, $w_{2}$ is the population, $c$ is the growth rate of the resources, $K$ the carrying capacity, $h$ the harvesting constant, and $a$ the growth rate of the population. More details on the construction of this model can be found in [10] where numerical solutions of the system (1)-(2) were given and a qualitative analysis of the general behavior of solutions both in finite and infinite time was presented.

In [11], we have applied Lie group analysis to this system and found that it can be integrated by quadrature if the involved parameters satisfy certain relationships. We have also discerned hidden linearity. Moreover, we have determined a Jacobi last multiplier and consequently a Lagrangian for the general system [12], and have found other cases, independent of and dependent on symmetry considerations in order to construct a corresponding variational problem, thus enabling us to find conservation laws by means of Noether's theorem. A comparison with the qualitative analysis given in [10] was provided.

In the present paper, we perform the quantization of the system (1)-(2); namely, we derive the Schrödinger equation by means of the method that preserves the Noether symmetries [13].

Moreover, in the wake of Volterra's last papers [14,15], the same mathematical model (1)-(2) is transformed into two second-order Lagrangian equations, and the superintegrability of this system is proven; namely three conservation laws, including the Hamiltonian, are found by means of Noether's theorem.

\section{Quantizing with Noether Symmetries}

It has been known for sixty-five years that quantization and nonlinear canonical transformations have no guarantee of consistency [16]. For a more recent perspective see [17] where an up-to-date account of the various approaches to tackle canonical transformation is also provided.

In $[13,18]$, a procedure which obviates the constraint imposed by the conflict between consistent quantization and the invariance of the Hamiltonian description under nonlinear canonical transformation was proposed. It is based on the preservation of Noether symmetries when going from classical to quantum mechanics. The quantization of classical problems is achieved by constructing a suitable time-dependent Schrödinger equation.

This method was reformulated in [19] for problems that are linearizable by Lie point symmetries, and also successfully applied to various classical problems: second-order Riccati equation [20], dynamics of a charged particle in a uniform magnetic field and a non-isochronous Calogero's goldfish system [18], an equation related to a Calogero's goldfish equation [21], two nonlinear equations somewhat related to the Riemann problem [22], a Liénard I nonlinear oscillator [19], a family of Liénard II nonlinear oscillators [23], $N$ planar rotors and an isochronous Calogero's goldfish system [24], the motion of a free particle and that of a harmonic oscillator on a double cone [25].

If a system of second-order equations is considered, i.e.,

$$
\ddot{\mathbf{x}}(t)=\mathbf{F}(t, \mathbf{x}, \dot{\mathbf{x}}), \quad \mathbf{x} \in \mathbb{R}^{N},
$$

that comes from a variational principle with a Lagrangian of first-order, then the quantization method that was first proposed in [13] consists of the following steps:

Step I. Find the Lie symmetries of the Lagrange equations

$$
\mathrm{Y}=W(t, \mathbf{x}) \partial_{t}+\sum_{k=1}^{N} W_{k}(t, \mathbf{x}) \partial_{x_{k}}
$$

Step II. Among them, find the Noether symmetries

$$
\Gamma=V(t, \mathbf{x}) \partial_{t}+\sum_{k=1}^{N} V_{k}(t, \mathbf{x}) \partial_{x_{k}} .
$$


This may require searching for the Lagrangian yielding the maximum possible number of Noether symmetries [26-29].

Step III. Construct the Schrödinger equation, where we assume $\hbar=1$ without loss of generality, admitting these Noether symmetries as Lie symmetries, namely

$$
2 i \psi_{t}+\sum_{k, j=1}^{N} f_{k j}(\mathbf{x}) \psi_{x_{j} x_{k}}+\sum_{k=1}^{N} h_{k}(\mathbf{x}) \psi_{x_{k}}+f_{0}(\mathbf{x}) \psi=0
$$

admitting the Lie symmetries

$$
\Omega=V(t, \mathbf{x}) \partial_{t}+\sum_{k=1}^{N} V_{k}(t, \mathbf{x}) \partial_{x_{k}}+G(t, \mathbf{x}, \psi) \partial_{\psi}
$$

without adding any other symmetries apart from the two symmetries that are present in any linear homogeneous partial differential equation, namely

$$
\psi \partial_{\psi}, \quad \alpha(t, \mathbf{x}) \partial_{\psi},
$$

where $\alpha=\alpha(t, \mathbf{x})$ is any solution of the Schrödinger Equation (4).

In [11], we derived $w_{1}$ from Equation (2), i.e.,

$$
w_{1}=-\frac{a u^{2}}{\dot{u}-a u} .
$$

and consequently obtained a second-order ordinary differential equation in $u \equiv w_{2}$,

$$
\ddot{u}=\frac{(2 a-h) \dot{u}^{2}}{a u}-(a+c-2 h) \dot{u}-\frac{a c}{K} u^{2}+a(c-h) u .
$$

Among other cases, we found that if the following relationships among the parameters $a, h$ and $c$ are satisfied

$$
a=2 c, \quad h=\frac{3}{2} c
$$

then Equation (6), i.e.,

$$
\ddot{u}=\frac{5 \dot{u}^{2}}{4 u}-\frac{2 c^{2}}{K} u^{2}-c^{2} u
$$

admits a three-dimensional Lie symmetry algebra generated by

$$
\Gamma_{1}=\partial_{t}, \quad \Gamma_{2}=e^{-c t}\left(\partial_{t}+2 c u \partial_{u}\right) \quad \Gamma_{3}=e^{c t}\left(\partial_{t}-2 c u \partial_{u}\right) .
$$

In [11], we proved that these three operators generate a representation of the complete symmetry group of Equation (8), determined a straightforward Jacobi last multiplier, i.e., [11]

$$
M=\frac{\sqrt{u}}{2 c u^{3}} .
$$

and consequently the following Lagrangian

$$
L=\sqrt{u}\left(\frac{\dot{u}^{2}}{4 c u^{3}}+\frac{c}{u}-\frac{2 c}{K}\right)
$$

that admits (9) as Noether symmetries. The three symmetries (9) are a representation of the complete symmetry group of Equation (8), namely a group that completely specifies a given differential equation 
through its algebraic representation [30]. Indeed, if we impose to the following general second-order ordinary differential equation

$$
\ddot{u}=F(t, u, \dot{u})
$$

to admit the symmetry algebra with generators (9), then we obtain the equation

$$
\ddot{u}=\frac{5 \dot{u}^{2}}{4 u}-B u^{2}-c^{2} u,
$$

namely a family of equations characterized by the parameter $B$, which can be replaced with $-2 c^{2} / K$ without any loss of generality.

We remark that it is important to have a Lagrangian, otherwise the quantization of Equation (8) cannot be pursued [31].

The Hamiltonian corresponding to the Lagrangian (11) is

$$
H=c \sqrt{u}\left(u^{2} p^{2}-\frac{1}{u}+\frac{2}{K}\right) .
$$

In the present paper, we quantize Equation (8), namely we derive the Schrödinger equation by preserving the Noether symmetries. We follow the three steps given above, i.e.,

Step I. We have found the three Lie symmetries, i.e., (9).

Step II. Those symmetries are also the Noether symmetries of the Lagrangian (11).

Step III. We consider the general equation

$$
2 i \Psi_{t}+f_{11}(u) \Psi_{u u}+h_{1}(u) \Psi_{u}+f_{0}(u) \Psi=0
$$

and impose that it should admit the following three Lie symmetries

$$
\Omega_{1}=\Gamma_{1}+G_{1}(t, u, \Psi) \partial_{\Psi}, \Omega_{2}=\Gamma_{2}+G_{2}(t, u, \Psi) \partial_{\Psi}, \Omega_{3}=\Gamma_{3}+G_{3}(t, u, \Psi) \partial_{\Psi},
$$

without adding any other symmetries apart from the two symmetries that are present in any linear homogeneous partial differential equation, namely

$$
\Psi \partial_{\Psi}, \quad \alpha(t, u) \partial_{\Psi},
$$

where $\alpha=\alpha(t, u)$ is any solution of the Schrödinger Equation (15).

Using ad hoc REDUCE interactive programs [32], we derive the following Schrödinger equation:

$$
2 i \Psi_{t}+u^{2} \sqrt{u} \Psi_{u u}-\sqrt{u}\left(\eta+4 \frac{c^{2}}{u}\right) \Psi=0,
$$

with $\eta$ any arbitrary constant.

Indeed, Equation (17) admits a Lie symmetry algebra generated by the following operators:

$$
\begin{aligned}
& \Omega_{1}=\Gamma_{1}, \\
& \Omega_{2}=\Gamma_{2}+c e^{-c t}\left(\frac{3}{2}+4 i \frac{c}{\sqrt{u}}\right) \Psi \partial_{\Psi} \\
& \Omega_{3}=\Gamma_{3}+c e^{c t}\left(-\frac{3}{2}+4 i \frac{c}{\sqrt{u}}\right) \Psi \partial_{\Psi} .
\end{aligned}
$$


The spectrum of this Schrödinger equation is continuous. This is not a surprise. In fact, in [11] we have derived that the general solution of Equation (8) is of exponential type, i.e.,

$$
u=\frac{64 A_{2}^{2} K}{\exp (2 c t)\left(8+A_{1}^{2} K+4 A_{2}^{2} K \exp (-2 c t)+4 A_{1} A_{2} K \exp (-c t)\right)^{2}},
$$

with $A_{1}, A_{2}$ arbitrary constants.

However, if we replace $t$ with $\tau=i t$, then Equation (8) becomes:

$$
\frac{\mathrm{d}^{2} u}{\mathrm{~d} \tau^{2}}=\frac{5}{4 u}\left(\frac{\mathrm{d} u}{\mathrm{~d} \tau}\right)^{2}+\frac{2 c^{2}}{K} u^{2}+c^{2} u
$$

This equation is one of the isochronous Liénard II equations [33], $\forall h=h(u)$, i.e.,

$$
\frac{\mathrm{d}^{2} u}{\mathrm{~d} \tau^{2}}+\frac{h^{\prime \prime}}{h^{\prime}}\left(\frac{\mathrm{d} u}{\mathrm{~d} \tau}\right)^{2}+\omega^{2} \frac{h}{h^{\prime}}+\frac{A}{h^{\prime} h^{3}}=0,
$$

with the identification $c^{2}=4 \omega^{2}$, and $h(u)=\left(A K /\left(2 \omega^{2} u\right)\right)^{1 / 4}$. The quantization of Equation (21) was given in [34]. In [23], the quantization that preserves the Noether symmetries was applied to all equations (21). Equation (20) gives rise to the following Schrödinger equation

$$
2 i \psi_{\tau}+\frac{\psi_{u u}}{\left(h^{\prime}\right)^{2}}-\frac{h^{\prime \prime} \psi_{u}}{\left(h^{\prime}\right)^{3}}+\left(\frac{A}{h^{2}}-\omega^{2} h^{2}\right) \psi=0 .
$$

Its eigenfunctions are:

$$
\psi_{n}=h^{\frac{k+1}{2}} e^{-i \frac{k+2}{2} \omega t-\frac{\omega}{2} h^{2}} L_{n}^{k / 2}\left(\omega h^{2}\right),
$$

with $k=\sqrt{1-4 A}$, and $L_{n}^{k / 2}$ the associated Laguerre polynomials, while the energy eigenvalues are:

$$
E_{n}=2 \omega\left(n+\frac{1}{2}+\frac{k}{4}\right) .
$$

More details can be found in [23].

\section{In the Wake of Volterra: A Superintegrable System}

In 1939 Volterra wrote [15]:

"I have been able to show that the equations of the struggle for existence depend on a question of Calculus of Variations (omissis). In order to obtain this result, I have replaced the notion of population by that of quantity of life [14]. In this manner I have also obtained some results by which dynamics is brought into relation to problems of the struggle for existence."

The quantity of life $X$ and the population $N$ of a species are connected by the relation

$$
N=\frac{\mathrm{d} X}{\mathrm{~d} t} .
$$

It is immediately obvious that this idea of raising the order of each equation is totally different from that by Trubach and Franco [35], who provided a method for finding a linear Lagrangian for systems of first-order equations. Also, Volterra's method is different from that of deriving a single second-order equation from a system of two first-order equations: indeed, Volterra takes a system of first-order equations and transforms it into a system of second-order equations.

In [12], Volterra's examples were analyzed and their connection to the Jacobi last multiplier was shown. 
Let us apply Volterra's method to the system (1)-(2).

We mimic Volterra by introducing what we call the quantity of natural life $u_{2}$, such that:

$$
w_{2}=\frac{\mathrm{d} u_{2}}{\mathrm{~d} t}
$$

Then, Equation (1) becomes a linear differential form in $w_{1}$ and $u_{2}$, and the substitution $w_{1}=r_{1}-h u_{2}$ simplifies it. Consequently, a Riccati equation is obtained, and the following linearizing transformation

$$
r_{1}=\frac{K}{c} \frac{\mathrm{d} u_{1}}{\mathrm{~d} t}
$$

yields

$$
\ddot{u}_{1}=\frac{c}{K^{2}}\left(K\left(2 h u_{2}+K\right) \dot{u}_{1}-h c\left(h u_{2}+K\right) u_{2} u_{1}\right),
$$

while Equation (2) becomes:

$$
\ddot{u}_{2}=\frac{a \dot{u}_{2}}{K \dot{u}_{1}-h c u_{2} u_{1}}\left(K \dot{u}_{1}-h c u_{2} u_{1}-c u_{1} \dot{u}_{2}\right) .
$$

Next, we check whether the system (28)-(29) admits a Lagrangian by applying Douglas's method [36], which consists of determining whether (at least) a Lagrangian exists for systems of two second-order ordinary differential equations that satisfy given conditions. We found that a Lagrangian (and only one) exists if $a=h=c$, namely if the system (28)-(29) becomes the following:

$$
\begin{aligned}
& \ddot{u}_{1}=\frac{c^{2} u_{2}}{K^{2}}\left(2 K \dot{u}_{1}-c^{2} u_{1} u_{2}\right)-\frac{c}{K}\left(c^{2} u_{1} u_{2}-K \dot{u}_{1}\right), \\
& \ddot{u}_{2}=c \dot{u}_{2}+\frac{c^{2} u_{1} \dot{u}_{2}^{2}}{c^{2} u_{1} u_{2}-K \dot{u}_{1}} .
\end{aligned}
$$

In [11], the case $a=h=c$ was discussed in Remark 3 of Section 4 where the general solution of $w_{2}$ in (1)-(2) was determined in implicit form, i.e.,

$$
\frac{A_{1}-3\left(c^{2}+1\right) u}{3 u \sqrt{u}}+\sqrt{\frac{K}{2 A_{1}}} \log \left(\frac{K}{A_{1} u}\right)+\sqrt{\frac{2 K}{A_{1}}} \log \left(A_{1}+\sqrt{A_{1}\left(A_{1}-c^{2} u\right)}\right)=t+A_{2} .
$$

The unique Lagrangian is

$$
L=2 K \log \left(c^{2} u_{1} u_{2}-K \dot{u}_{1}\right) \dot{u}_{2}+c u_{2}\left(c u_{2}+2 K\right) .
$$

It admits three Noether symmetries generated by the following operators:

$$
\Gamma_{1}=\partial_{t}, \quad \Gamma_{2}=c^{2} t u_{1} \partial_{u_{1}}+K \partial_{u_{2}}, \quad \Gamma_{3}=u_{1} \partial_{u_{1}} .
$$

The corresponding first integrals are:

$$
\begin{aligned}
& I_{1}=-c u_{2}\left(c u_{2}+2 K\right)-\frac{2 K^{2} \dot{u}_{1} \dot{u}_{2}}{c^{2} u_{1} u_{2}-K \dot{u}_{1}}, \\
& I_{2}=2 K \log \left(c^{2} u_{1} u_{2}-K \dot{u}_{1}\right)-2 c^{2} t u_{2}-2 c K t-\frac{2 c^{2} K t u_{1} \dot{u}_{2}}{c^{2} u_{1} u_{2}-K \dot{u}_{1}}, \\
& I_{3}=u_{2}+\frac{K u_{1} \dot{u}_{2}}{c^{2} u_{1} u_{2}-K \dot{u}_{1}},
\end{aligned}
$$

with gauge functions $F_{1}=0, F_{2}=2 c K t\left(c u_{2}+K\right)$, and $F_{3}=u_{2}$, respectively. 
The first integral $I_{1}$ in (34) is the Hamiltonian. Indeed, if one introduces the momenta $p_{1}, p_{2}$ by means of the Legendre transformation applied to the Lagrangian (32), i.e.,

$$
\begin{aligned}
& p_{1} \equiv \frac{\partial L}{\partial \dot{u}_{1}}=-\frac{2 K^{2} \dot{u}_{2}}{c^{2} u_{1} u_{2}-K \dot{u}_{1}}, \\
& p_{2} \equiv \frac{\partial L}{\partial \dot{u}_{2}}=2 K \log \left(c^{2} u_{1} u_{2}-K \dot{u}_{1}\right)
\end{aligned}
$$

then the corresponding Hamiltonian, i.e. $H \equiv p_{1} \dot{u}_{1}+p_{2} \dot{u}_{2}-L$, is

$$
H=-c u_{2}\left(c u_{2}+2 K\right)+\frac{1}{K}\left(c^{2} p_{1} u_{1} u_{2}-p_{1} \exp \left(\frac{p_{2}}{2 K}\right)\right),
$$

that is $I_{1}$ in (34), if $\dot{u}_{1}, \dot{u}_{2}$ are replaced with $p_{1}, p_{2}$, as given in (35).

Introducing the momenta into $I_{2}$, and $I_{3}$ in (34) yields:

$$
\begin{aligned}
& \text { Int }_{2}=p_{2}-2 c^{2} t u_{2}-2 c K t+\frac{c^{2} t p_{1} u_{1}}{K} \\
& \text { Int }_{3}=u_{2}-\frac{p_{1} u_{1}}{2 K}
\end{aligned}
$$

respectively. Then the Hamiltonian system is superintegrable, since we have been able to determine three first integrals.

\section{Discussion and Final Remarks}

We have shown that it is possible to quantize and also detect superintegrability of biological models. The key is to find a Lagrangian and its admitted Noether symmetries. The Easter Island model (1)-(2) was used as an example. Both classical quantization and superintegrability could be achieved if the involved parameters satisfy certain relationships, namely $a=2 c, h=\frac{3}{2} c$, and $a=h=c$, respectively.

Classical quantization was achieved by applying the quantization method that preserves the Noether symmetries [13]. We have derived the Schrödinger equation corresponding to Equation (8) by preserving the three Noether symmetries (9). Also, we have shown that if we replace the independent variable $t$ with $\tau=i t$, then Equation (8) is transformed into Equation (20), which is one of the isochronous Liénard II equations [33]. Its corresponding Schrödinger equation was derived in [23,34]. The eigenfunctions and energy eigenvalues are given in (23) and (24), respectively.

About superintegrability, we have applied Volterra's idea [14] to system (1)-(2) by introducing the quantity of natural life $u_{2}$ as given in (26), and therefore increasing the order of the system (1)-(2). Consequently, a Riccati equation also appeared and we have applied the known transformation (27) that yields a second-order equation. Thus, we have obtained a system of two second-order equations (28) and (29). Then, we have applied Douglas's method and derived a unique Lagrangian if the involved parameters satisfy the relationships $a=h=c$. Finally, we have shown that the corresponding Hamiltonian (36) yields a superintegrable Hamiltonian system, since we have been able to determine three first integrals (34) by means of Noether's theorem.

We would like to conclude with the following statement in [37]:

"Only rarely does one find mention, at post-graduate level, of any problem in connection with the process of actually solving such equations. The electronic computer may perhaps be partly to blame for this, since the impression prevails in many quarters that almost any differential equation problem can be merely put on the machine, so that finding an analytical solution is largely a waste of time. This, however, is only a small part of the truth, for at higher levels there are generally so many parameters or boundary conditions involved that numerical solutions, even if practicable, give no real idea of the properties of the equation. Moreover, any analyst of sensibility will feel that to fall back on numerical 
techniques savours somewhat of breaking a door with a hammer when one could, with a little trouble, find the key".

Acknowledgments: M.C.N. acknowledges the support of University of Perugia through Fondi Ricerca di base 2015 (years 2016-2017).

Author Contributions: The authors contributed equally to this work.

Conflicts of Interest: The authors declare no conflict of interest.

\section{References}

1. Krakauer, D.C.; Collins, J.P.; Erwin, D.; Flack, J.C.; Fontana, W.; Laubichler, M.D.; Prohaska, S.J.; West, G.B.; Stadler, P.F. The challenges and scope of theoretical biology. J. Theor. Biol. 2011, 276, 269-276.

2. Rowe, D. Einstein meets Hilbert: At the Crossroads of Physics and Mathematics. Phys. Perspect. 2001, 3, 379-424.

3. Nucci, M.C. Using Lie symmetries in epidemiology. Electron. J. Differ. Equ. 2004, 12, 87-101.

4. Torrisi, V.; Nucci, M.C. Application of Lie group analysis to a mathematical model which describes HIV transmission. In The Geometrical Study of Differential Equations; Leslie, J.A., Hobart, T.P., Eds.; American Mathematical Society: Providence, RI, USA, 2001; pp. 11-20.

5. Nucci, M.C.; Leach, P.G.L. An integrable S-I-S model. J. Math. Anal. Appl. 2004, 290, 506-518.

6. Edwards, M.; Nucci, M.C. Application of Lie group analysis to a core group model for sexually transmitted diseases. J. Nonlinear Math. Phys. 2006, 13, 211-230.

7. Gradassi, A.; Nucci, M.C. Hidden linearity in systems for competition with evolution in ecology and finance. J. Math. Anal. Appl. 2007, 333, 274-294.

8. Nucci, M.C.; Leach, P.G.L. Lie integrable cases of the simplified multistrain/two-stream model for tuberculosis and Dengue fever. J. Math. Anal. Appl. 2007, 333, 430-449.

9. Freeman, J.D. Missed opportunities. Bull. Am. Math. Soc. 1972, 78, 635-652.

10. Basener, B.; Ross, D.S. Booming and crashing populations and Easter Island. SIAM J. Appl. Math. 2005, 65, 684-701.

11. Nucci, M.C.; Sanchini, G. Symmetries, Lagrangians and Conservation Laws of an Easter Island Population Model. Symmetry 2015, 7, 1613-1632.

12. Nucci, M.C.; Tamizhmani, K.M. Lagrangians for biological models. J. Nonlinear Math. Phys. 2012, $19,1250021$.

13. Nucci, M.C. Quantization of classical mechanics: Shall we Lie? Theor. Math. Phys. 2011, 168, $994-1001$.

14. Volterra, V. Population growth, equilibria, and extinction under specified breeding conditions: A development and extension of the theory of the logisitc curve. Hum. Biol. 1938, 10, 1-11.

15. Volterra, V. Calculus of Variations and the Logistic Curve. Hum. Biol. 1939, 11, 173-178.

16. Van Hove, L. Sur certaines représentations unitaires d'un groupe infini de transformations. Memoires Acad. R. Belg. Cl. Sci. 1951, 26, 1-102.

17. Błaszak, M.; Domański, Z. Canonical transformations in quantum mechanics. Ann. Phys. 2013, 331, 70-96.

18. Nucci, M.C. Quantizing preserving Noether symmetries. J. Nonlinear Math. Phys. 2013, 20, 451-463.

19. Gubbiotti, G.; Nucci, M.C. Noether symmetries and the quantization of a Liénard-type nonlinear oscillator. J. Nonlinear Math. Phys. 2014, 21, 248-264.

20. Nucci, M.C. From Lagrangian to Quantum Mechanics with Symmetries. J. Phys. Conf. Ser. 2012, 380, 012008.

21. Nucci, M.C. Symmetries for thought. Math. Notes Miskolc 2013, 14, 461-474.

22. Nucci, M.C. Spectral realization of the Riemann zeros by quantizing $H=w(x)\left(p+\ell_{p}^{2} / p\right)$ : The Lie-Noether symmetry approach. J. Phys. Conf. Ser. 2014, 482, 012032.

23. Gubbiotti, G.; Nucci, M.C. Quantization of quadratic Liénard-type equations by preserving Noether symmetries. J. Math. Anal. Appl. 2015, 422, 1235-1246.

24. Nucci, M.C. Ubiquitous symmetries. Theor. Math. Phys. 2016, 188, 1361-1370.

25. Gubbiotti, G.; Nucci, M.C. Quantization of the dynamics of a particle on a double cone by preserving Noether symmetries. arXiv 2016, arXiv:1607.00543.

26. Nucci, M.C.; Leach, P.G.L. Lagrangians galore. J. Math. Phys. 2007, 48, 123510.

27. Nucci, M.C.; Leach, P.G.L. Jacobi last multiplier and Lagrangians for multidimensional linear systems. J. Math. Phys. 2008, 49, 073517. 
28. Nucci, M.C.; Tamizhmani, K.M. Using an old method of Jacobi to derive Lagrangians: A nonlinear dynamical system with variable coefficients. Nuovo Cimento B 2010, 125, 255-269.

29. Nucci, M.C.; Tamizhmani, K.M. Lagrangians for dissipative nonlinear oscillators: The method of Jacobi Last Multiplier. J. Nonlinear Math. Phys. 2010, 17, 167-178.

30. Krause, J. On the complete symmetry group of the classical Kepler system. J. Math. Phys. 1994, 35, 5734-5748.

31. Hojman, S.A.; Shepley, L.C. No Lagrangian? No quantization! J. Math. Phys. 1991, 32, 142-146.

32. Nucci, M.C. Interactive REDUCE programs for calculating Lie point, non-classical, Lie-Bäcklund, and approximate symmetries of differential equations: Manual and floppy disk. In CRC Handbook of Lie Group Analysis of Differential Equations. Vol. 3: New Trends in Theoretical Developments and Computational Methods; Ibragimov, N.H., Ed.; CRC Press: Boca Raton, FL, USA, 1996; pp. 415-481.

33. Tiwari, A.K.; Pandey, S.N.; Senthilvelan, M.; Lakshmanan, M. Classification of Lie point symmetries for quadratic Liénard type equation $\ddot{x}+f(x) \dot{x}^{2}+g(x)=0$. J. Math. Phys. 2013, 54, 053506.

34. Choudhury, A.G.; Guha, P. Quantization of the Liénard II equation and Jacobi's last multiplier. J. Phys. A Math. Theor. 2013, 46, 165202.

35. Trubatch, S.L.; Franco, A. Canonical Procedures for Population Dynamics. J. Theor. Biol. 1974, 48, $299-324$.

36. Douglas, J. Solution of the Inverse Problem of the Calculus of Variations. Trans. Am. Math. Soc. 1941, 50, 71-128.

37. Arscott, F.M. Periodic Differential Equations; Pergamon Press: Oxford, UK, 1964.

(C) 2016 by the authors; licensee MDPI, Basel, Switzerland. This article is an open access article distributed under the terms and conditions of the Creative Commons Attribution (CC-BY) license (http://creativecommons.org/licenses/by/4.0/). 